\title{
Ideação suicida, tentativa de suicídio ou suicídio em adolescentes: revisão narrativa
}

\author{
Suicidal ideation, suicide attempt or suicide in adolescents: narrative review \\ Ideación suicida, intento de suicidio o suicidio en adolescentes: revisión narrativa
}

Francieli Franco Soster

ORCID: https://orcid.org/0000-0002-1634-0195

Universidade Federal de Santa Maria, Brasil

E-mail: francielifs.com@gmail.com

Andressa da Silveira

ORCID: https://orcid.org/0000-0002-4182-4714

Universidade Federal de Santa Maria, Brasil E-mail: andressadasilveira@gmail.com

Gabriélli Maria Huppes

ORCID: https://orcid.org/0000-0001-9019-0669

Universidade Federal de Santa Maria, Brasil E-mail: gabriellihuppes@gmail.com

Leila Mariza Hildebrandt

ORCID: https://orcid.org/0000-0003-0504-6166

Universidade Federal de Santa Maria, Brasil

E-mail: leilahildebrandt@yahoo.com.br

Fernanda Beheregaray Cabral

ORCID: https://orcid.org/0000-0002-4809-278X

Universidade Federal de Santa Maria, Brasil E-mail: cabralfernandab@gmail.com

Regina Gema Santini Costenaro

ORCID: https://orcid.org/0000-0001-8657-2066

Universidade Franciscana, Brasil

E-mail: reginacostenaro@gmail.com

\begin{abstract}
Resumo
Objetivo: caracterizar o que tem sido produzido acerca dos fatores de risco para a ideação suicida, tentativa de suicídio ou suicídio em adolescente. Método: revisão narrativa de literatura acerca dos fatores de risco para ideação suicida, tentativa de suicídio ou suicídio em adolescentes. A busca foi realizada de março a julho de 2020 com os descritores "Adolescentes" [AND] "Suicídio" [AND] "Fatores de risco". Foram incluídas publicações de artigos disponibilizados na íntegra, nos idiomas português, inglês e espanhol, com espaço temporal de cinco anos. Para a etapa de levantamento bibliográfico foram utilizadas as bases Literatura Latino-Americana e do Caribe de Informações em Ciências da Saúde (LILACS), a National Library of Medicine (PubMed) e a Scientific Electronic Library Online (SciELO), totalizando 25 artigos que compõem o corpo desta pesquisa, após a aplicação dos critérios de inclusão e exclusão. Resultados: o suicídio é multifatorial e encontra-se correlacionado com aspectos sociais, biológicos e psicológicos de cada adolescente, em que questões relacionadas à exposição à violência, relações familiares conturbadas, acontecimentos de vida negativos, desigualdades sociais, problemas educacionais, depressão e uso de drogas lícitas e ilícitas atuam como fatores de risco para o suicídio em adolescentes. Conclusão: os adolescentes se encontram vulneráveis a diversos agravos que podem fragilizar sua saúde mental, o que somado à influência negativa dos fatores de risco de origem biológica, econômica, psicológica e social, atuam como agentes causadores do desenvolvimento de ideações suicidas e/ou tentativas de suicídio.
\end{abstract}

Palavras-chave: Adolescentes; Suicídio; Fatores de risco; Saúde mental.

\begin{abstract}
Objetive: Characterize what has been produced about risk factors for suicidal ideation, suicide attempt or suicide in the adolescent. Method: Narrative literature review about risk factors for suicidal ideation, suicide attempt or suicide in adolescents. The research was carried out from March to July 2020 with the descriptors "Adolescents" [AND] "Suicide" [AND] "Risk factors". Publications of articles made available in full were included, in Portuguese, English and Spanish, with a five-year timeframe. For the bibliographic survey stage Latin American and Caribbean Health Sciences Information bases were used (LILACS), the National Library of Medicine (PubMed) and Scientific Electronic Library Online (SciELO). Totaling 25 articles that make up the body of this research, after applying the inclusion and exclusion criteria. Results: Suicide is multifactorial and is correlated with social aspects, biological and psychological aspects of each adolescent, where issues related to exposure to violence, troubled family relationships, negative life events, social diferences, educational problems, depression and use of legal and illegal drugs act as risk
\end{abstract}


factors for suicide in adolescents. Conclusion: Adolescents are vulnerable to several problems that can weaken their mental health, which added to the negative influence of risk factors of biological origin, economical, psychological and social, act as causative agents that cause the development of suicidal ideas and/or suicide attempts.

Keywords: Adolescents; Suicide; Risk factors; Mental health.

\section{Resumen}

Objetivo: caracterizar lo producido sobre factores de riesgo de ideación suicida, intento de suicidio o suicidio en el adolescente. Método: revisión de la literatura narrativa sobre factores de riesgo de ideación suicida, intento de suicidio o suicidio en adolescentes. La investigación se realizó de marzo a julio de 2020 con los descriptores "Adolescentes" [Y] "Suicidio" [Y] "Factores de riesgo". Se incluyeron publicaciones de artículos completos, en portugués, inglés y español, con un plazo de cinco años. Para la etapa de relevamiento bibliográfico se utilizaron las Bases de Información de Ciencias de la Salud de América Latina y el Caribe (LILACS), la Biblioteca Nacional de Medicina (PubMed) y la Biblioteca Electrónica Científica en Línea (SciELO). En total 25 artículos que conforman el cuerpo de esta investigación, luego de aplicar los criterios de inclusión y exclusión. Resultados: El suicidio es multifactorial y se correlaciona con aspectos sociales, biológicos y psicológicos de cada adolescente, donde se encuentran temas relacionados con la exposición a la violencia, relaciones familiares conflictivas, eventos de vida negativos, diferencias sociales, problemas educativos, depresión y uso de drogas legales e ilegales actúan como factores de riesgo de suicidio en adolescentes. Conclusión: Los adolescentes son vulnerables a diversos problemas que pueden debilitar su salud mental, los cuales sumados a la influencia negativa de factores de riesgo de origen biológico, económico, psicológico y social, actúan como agentes causales que provocan el desarrollo de ideas suicidas y/o intentos de suicidio.

Palabras clave: Adolescentes; Suicidio; Factores de riesgo; Salud mental.

\section{Introdução}

A Organização Mundial da Saúde (OMS) define que a faixa etária da adolescência compreende dos 10 aos 19 anos de idade (OMS, 2014). Já o Estatuto da Criança e do Adolescente (ECA), por meio da Lei no 8.069 de 1990, definiu que a adolescência corresponde à faixa etária dos 12 aos 18 anos idade (Brasil, 1990). No entanto, percebe-se que o conceito de adolescência se transforma com as mudanças relacionadas à inserção social, tanto quanto ao contexto biopsicossocial, fazendo com que a experiência de adolescer seja singular a cada indivíduo (Rossi, et al., 2019; Senna \& Dessen, 2015).

$\mathrm{O}$ adolescer promove o desligamento de pensamentos originários na infância, estimulando os adolescentes a assumirem responsabilidades que auxiliam no seu desenvolvimento cognitivo e comportamental. Tais transformações resultam em um processo de formação psicossocial em que o adolescente desenvolve os pilares essenciais da sua personalidade e valores morais (Gadêlha \& Gonçalves, 2017). Esta etapa é marcada por um comportamento questionador, impulsivo e inseguro, o que pode resultar em gatilhos para o desenvolvimento de problemas, com comprometimento da saúde mental (Silva, et al., 2015).

A saúde mental de adolescentes é uma temática extremamente estigmatizada, o que resulta no afastamento destes indivíduos dos serviços de saúde (Silva, et al., 2019). A exposição de adolescentes aos fatores de risco, somada à dificuldade dessa população em acessar serviços de saúde, resulta na falta de assistência aos adolescentes, tornando-os vulneráveis a diversos agravos, dentre eles, as ideações suicidas (Braga \& Marques \& Alves, 2017).

A ideação suicida no adolescente torna-se patológica quando a intensidade e recorrência desses pensamentos aumentam, fazendo com que o indivíduo veja o suicídio como a única saída para suas perturbações. As tentativas de suicídio são consideradas agravos de notificação compulsória, são atos intencionais de autoagressão que não resultam em morte, sendo um preditor para o suicídio (Abreu \& Souza, 2017; Sadock \& Sadock, 2017). E o suicídio é um ato deliberado do indivíduo de pôr fim à própria vida, esse fenômeno inclui desde pensamentos/ideações suicidas, até mesmo, planejamento e tentativas de autodestruição que podem resultar na ação de suicidar-se (Couto \& Tavares, 2016).

O suicídio é a segunda principal causa de morte dos jovens e adultos entre 15 a 29 anos de idade, sendo que a cada suicídio consumado ocorrem uma média de 10 a 20 tentativas de suicídio. No Brasil, a taxa de suicídios entre jovens cresceu em média 30\% nos últimos 25 anos (OMS, 2016; Ricci, et al., 2016). Entre 2011 a 2016 foram registrados 48.204 casos de 
tentativa de suicídios, sendo 69\% em mulheres e 31\% em homens. Nesse período, constam registrados 55.649 óbitos por suicídio, chegando-se à taxa de 5,5/100 mil habitantes, sendo o risco de suicídio masculino de 8,7/100 habitantes e o feminino 2,4/100 mil habitantes (Ministério da Saúde, 2017).

Assim, este estudo apresenta como questão de pesquisa: "O que tem sido produzido na literatura científica acerca dos fatores de risco para a ideação suicida, tentativa de suicídio ou suicídio entre adolescentes?"

Frente ao exposto, este estudo objetiva caracterizar o que tem sido produzido acerca dos fatores de risco para a ideação suicida, tentativa de suicídio ou suicídio em adolescentes.

\section{Metodologia}

Estudo qualitativo, do tipo Revisão Narrativa da Literatura (RNL), o qual caracteriza-se por sua ampla abordagem, permitindo a descrição e caracterização de publicações, com a finalidade de analisar os achados e interpretá-los (Brum, et al., 2015). Estudos de RNL permitem a descrição e discussão do estado da arte de um determinado tema, sob ponto de vista teórico, a partir da análise dos temas abrangentes é possível identificar lacunas no conhecimento. As revisões narrativas podem contribuir no debate de determinadas temáticas, levantando temas e contribuem para a construção do conhecimento (Costa, et al., 2015).

O desenvolvimento desta revisão realizou-se inicialmente pela identificação do tema que, neste caso, é referente aos fatores de risco para a tentativa de suicídio e/ou suicídio entre a população de adolescentes. Logo após, foi delineada a pergunta de pesquisa, com o objetivo de alinhar a construção da RNL. "O que tem sido produzido na literatura científica acerca dos fatores de risco para a ideação suicida, tentativa de suicídio ou suicídio entre adolescentes? ” Posteriormente, foi definido o objetivo da pesquisa "identificar e caracterizar na produção científica fatores de risco para a ideação suicida, tentativa de suicídio ou suicídio na população adolescente".

Para etapa de levantamento bibliográfico e buscas, foram utilizadas as bases Literatura Latino-Americana e do Caribe de Informações em Ciências da Saúde (LILACS), a qual teve início a partir de uma iniciativa da Organização Pan-americana de Saúde (PAHOWHO), ligada à Organização das Nações Unidas. Atualmente abrange 26 países e 898 periódicos, sendo o mais importante índice da literatura técnico-científica da América Latina e Caribe (Castelar \& Daltro \& Barreto, 2019).

A National Library of Medicine (PubMed) se configura como um recurso gratuito que apoia a busca e recuperação da literatura biomédica e de ciências da vida. E, por fim, a Scientific Electronic Library Online (SciELO), que atua como um modelo para a publicação eletrônica cooperativa de periódicos científicos na internet. Especialmente desenvolvida para responder às necessidades da comunicação científica nos países em desenvolvimento e particularmente na América Latina e Caribe (Packer, et al. 1998).

Definiram-se como critérios de inclusão: artigos disponibilizados na íntegra, nos idiomas português, inglês e espanhol, com espaço temporal de cinco anos, sendo incluídas produções do ano de 2015 a 2019, devido à coleta de dados ter ocorrido no ano de 2020. Justifica-se esse período, pelo aumento das lesões autoprovocadas, as quais podem estar associadas a estratégias utilizadas por adolescentes que apresentam ideação e fatores de risco para o suicídio, ou então a formas de aliviar sofrimentos vivenciados. Os dados apresentam um crescimento de 17.704 casos de lesões autoprovocadas em adolescentes entre 10 a 19 anos no Brasil, passando de 8.939 em 2015, para 26.643 casos em 2018 (SINAN, 2020). Ademais, destaca-se a rapidez das informações veiculadas nas bases de dados e validade das produções para publicação científica.

Em relação aos critérios de exclusão, excluíram-se trabalhos de conclusão de curso, monografias, dissertações, teses, nota prévia, estudos de revisão, manuais, livros, e-books, artigos em que não houvesse a disponibilidade do material gratuito na íntegra e as produções que estivessem duplicadas.

O levantamento bibliográfico teve início em março de 2020 estendendo-se até julho do mesmo ano. Para a busca na 
SciELO, foram utilizados os descritores da lista DeCS, "Adolescentes" [AND] "Suicídio" [AND] "Fatores de risco", resultando em 10 artigos. O acesso à base de dados LILACS ocorreu por meio da Biblioteca Virtual em Saúde (BVS), onde foram usados os descritores encontrados em inglês na lista DeCS "Adolescent" [AND] "Suicide" [AND] "Risk factors", tendo como produto 48 artigos. Para a base de dados PubMed foram utilizados os descritores indexados no Medical Subject Headings (MESH), sendo eles "Adolescent" [AND] "Suicide" [AND] "Risk factors" onde foram obtidos 37 artigos, totalizando 95 publicações para leitura.

Estas foram filtradas pelos critérios de inclusão e de exclusão, obtendo-se 25 artigos após a leitura do resumo. Do total foram descartados seis artigos por serem repetidos, três na base de dados Scielo e três na Lilacs. Dessa forma, o corpus do estudo foi composto por 25 artigos conforme a descrição da Figura 1.

Figura 1 - Fluxograma de seleção da produção científica Scielo, Pubmed e Lilacs, 2020.

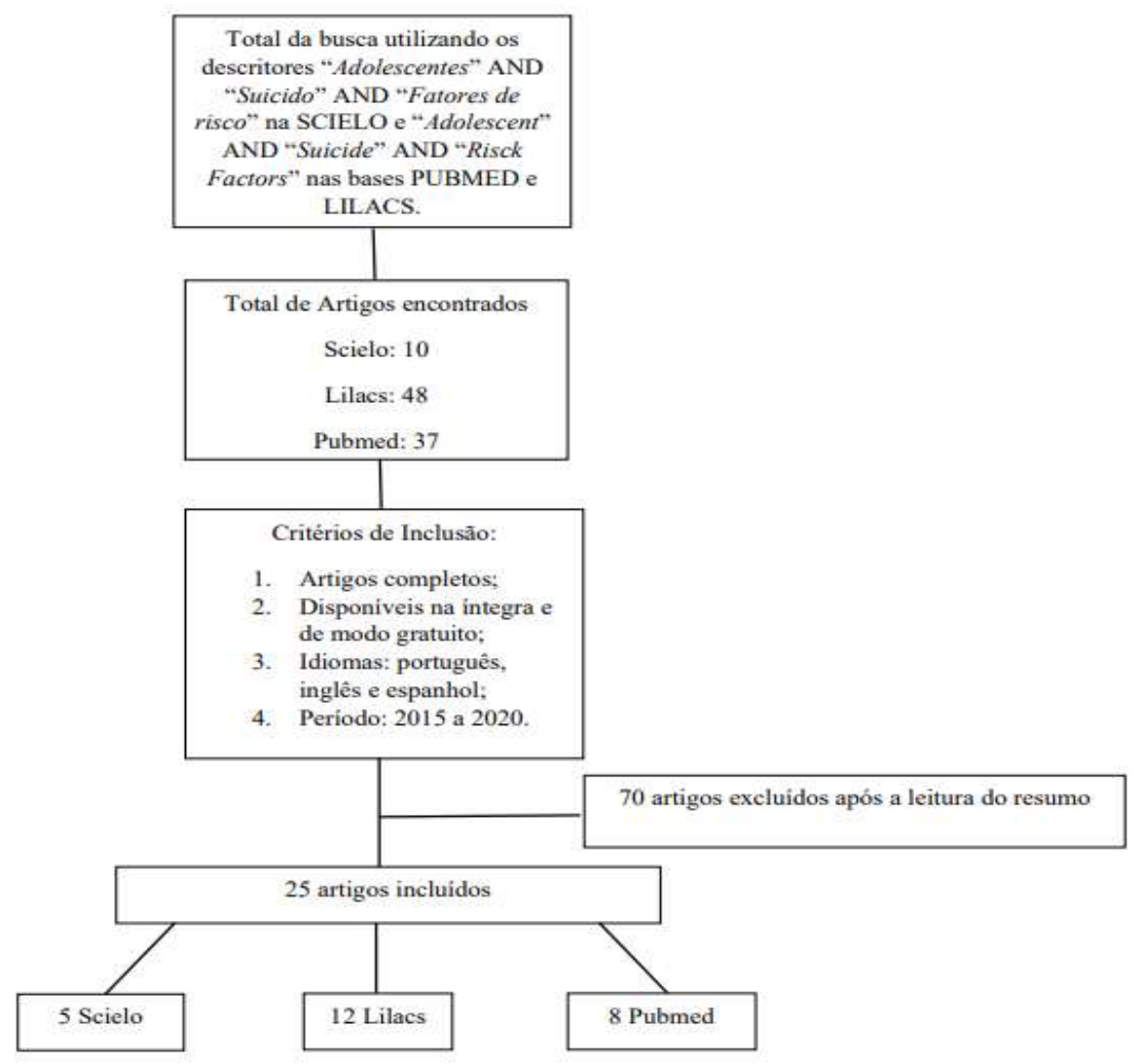

Fonte: Dados da Pesquisa (2020).

Após, as produções foram alocadas em um quadro sinóptico (Quadro 1) a fim de facilitar o processo de identificação da produção do conhecimento acerca do tema estudado, com as seguintes informações: código do artigo (A1, A2, A3...), título, ano de publicação, periódico e síntese dos resultados. Por fim, utilizou-se a leitura dos artigos selecionados e realizou-se a caracterização das publicações, extraindo informações que foram convergentes no decorrer deste processo.

\section{Resultados}

A caracterização do estudo de revisão narrativa será apresentada no quadro sinóptico (Quadro 1), em que constam as informações incluídas neste estudo. 
Quadro 1 - Caracterização da produção científica Scielo, Pubmed e Lilacs, 2020.

\begin{tabular}{|c|c|c|c|c|}
\hline Código & Título & $\begin{array}{c}\text { Ano de } \\
\text { Publicação }\end{array}$ & Periódico & Síntese dos Resultados \\
\hline A1 & $\begin{array}{l}\text { Epidemiología del } \\
\text { suicídio en } \\
\text { adolescentes y jóvenes } \\
\text { en Ecuador }\end{array}$ & 2018 & $\begin{array}{l}\text { Revista } \\
\text { Panamericana de } \\
\text { Salud Publica }\end{array}$ & $\begin{array}{l}\text { Entre } 2001 \text { e } 2014 \text {, houve } 4.855 \text { suicídios em } \\
\text { adolescentes e jovens. O maior risco foi estimado } \\
\text { em homens de } 15 \text { a } 24 \text { anos e em adolescentes } \\
\text { residentes na Amazônia, seguidos pelos da Serra. O } \\
\text { grupo étnico com mais casos de suicídio foi o de } \\
\text { mestiços, embora } 40 \% \text { dos jovens que cometem } \\
\text { suicídio na Amazônia sejam indígenas. O método } \\
\text { mais comum foi o enforcamento seguido de } \\
\text { envenenamento por pesticidas. }\end{array}$ \\
\hline $\mathrm{A} 2$ & $\begin{array}{l}\text { Riesgo de suicídio } \\
\text { adolescente } \\
\text { localidades urbanas y } \\
\text { rurales por género, } \\
\text { región de Coquimbo, } \\
\text { Chile }\end{array}$ & 2018 & Aquichan & $\begin{array}{l}\text { Não existem diferenças no risco de suicídio } \\
\text { segundo a localização geográfica, mas sim por } \\
\text { gênero; apresentaram-se } 15,76 \% \text { de adolescentes do } \\
\text { sexo feminino com risco suicida. }\end{array}$ \\
\hline A3 & $\begin{array}{l}\text { Adolescentes } \\
\text { riesgo: en } \\
\text { asociados con el } \\
\text { intento de suicídio en } \\
\text { México }\end{array}$ & 2017 & $\begin{array}{l}\text { Revista Gerencia y } \\
\text { Políticas de Salud }\end{array}$ & $\begin{array}{l}\text { As mulheres relataram maior prevalência de } \\
\text { tentativa de suicídio, sendo menor para os mais } \\
\text { jovens. Se ele foi agredido ou teve uma defasagem } \\
\text { educacional aumentou a propensão a tentar } \\
\text { suicídio, assim como aqueles adolescentes que } \\
\text { usam álcool ou tabaco. }\end{array}$ \\
\hline A4 & $\begin{array}{l}\text { Association between } \\
\text { symptoms of anorexia } \\
\text { and bulimia nervosa } \\
\text { and suicidal behavior } \\
\text { in school children of } \\
\text { Boyacá, Colombia }\end{array}$ & 2017 & $\begin{array}{l}\text { Acta Colombiana de } \\
\text { Psicología }\end{array}$ & $\begin{array}{l}\text { Os resultados mostraram que, do total de } \\
\text { participantes, } 17,8 \% \text { (230) pensam no suicídio } \\
\text { como alternativa e que } 42 \% \text { dos que registram } \\
\text { sintomatologia relacionada com transtornos } \\
\text { alimentares apresentam ideação negativa. }\end{array}$ \\
\hline A5 & 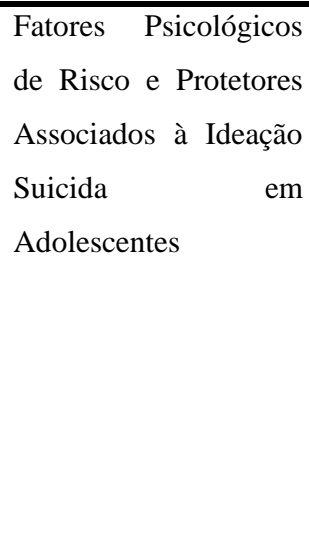 & 2016 & $\begin{array}{l}\text { Psicologia, Saúde \& } \\
\text { Doenças }\end{array}$ & $\begin{array}{l}\text { Os resultados caracterizam o contributo diferencial } \\
\text { de fatores de risco (acontecimentos de vida } \\
\text { negativos e desesperança) e de proteção (razões } \\
\text { para viver, autoestima e satisfação com o suporte } \\
\text { social), os quais, em conjunto, explicam cerca de } \\
40 \% \text { da ideação suicida. Verificou-se ainda, que os } \\
\text { níveis de ideação suicida são diretamente } \\
\text { influenciados pelos acontecimentos de vida } \\
\text { negativos, coexistindo com uma influência mediada } \\
\text { por fatores psicológicos. }\end{array}$ \\
\hline A6 & $\begin{array}{l}\text { Suicide mortality } \\
\text { among youth in } \\
\text { southern Brazil: a } \\
\text { spatiotemporal }\end{array}$ & 2019 & $\begin{array}{l}\text { Brazilian Journal of } \\
\text { Psychiatry }\end{array}$ & $\begin{array}{l}\text { Observou-se dependência espacial em taxas de } \\
\text { mortalidade por suicídio em ambos os períodos. Os } \\
\text { resultados mostram que a privação socioeconômica } \\
\text { no município foi um importante determinante para }\end{array}$ \\
\hline
\end{tabular}




\begin{tabular}{|c|c|c|c|c|}
\hline & $\begin{array}{l}\text { evaluation of } \\
\text { socioeconomic } \\
\text { vulnerability }\end{array}$ & & & $\begin{array}{l}\text { o suicídio na população jovem paranaense, e } \\
\text { influenciou significativamente a formação de } \\
\text { aglomerados de alto risco. }\end{array}$ \\
\hline A7 & $\begin{array}{l}\text { Intento de suicídio en } \\
\text { adolescentes: Factores } \\
\text { asociados }\end{array}$ & 2019 & $\begin{array}{l}\text { Revista chilena de } \\
\text { pediatría }\end{array}$ & $\begin{array}{l}\text { A prevalência estimada de tentativa de suicídio foi } \\
\text { de } 6,8 \% \text {. Os fatores que aumentam a chance de } \\
\text { tentativa de suicídio foram: ser mulher, ter menos } \\
\text { de } 16 \text { anos, viver em clima familiar ruim ou com } \\
\text { pouca comunicação com os pais, ter baixa estima, } \\
\text { problemas comportamentais ou de aprendizagem ou } \\
\text { ter uma doença mental; destacam-se ainda contato } \\
\text { sexual forçado; ou uso de drogas, tabaco ou álcool. }\end{array}$ \\
\hline A8 & $\begin{array}{l}\text { The association } \\
\text { between adolescent } \\
\text { suicide rates and } \\
\text { socioeconomic } \\
\text { indicators in Brazil: a } \\
\text { 10-year retrospective } \\
\text { ecological study }\end{array}$ & 2019 & $\begin{array}{l}\text { Brazilian Journal of } \\
\text { Psychiatry }\end{array}$ & $\begin{array}{l}\text { A taxa de suicídio de adolescentes aumentou } 24 \% \\
\text { ao longo do período de estudo. A desigualdade } \\
\text { social (avaliada pelo índice de Gini), esteve } \\
\text { positivamente associada às taxas globais de suicídio } \\
\text { de adolescentes. Após desagregar os achados por } \\
\text { idade (10-14 e 15-19 anos), a desigualdade social } \\
\text { esteve associada à taxa de suicídio apenas para } \\
\text { adolescentes de } 15 \text { a } 19 \text { anos. Desagregando esses } \\
\text { achados por sexo, a associação com as variáveis } \\
\text { econômicas tornou-se significativa apenas entre as } \\
\text { mulheres. Os homens apresentaram maior taxa } \\
\text { global de suicídio do que as do sexo feminino, e a } \\
\text { maior taxa foi observada em adolescentes do sexo } \\
\text { masculino de } 15 \text { a } 19 \text { anos. Níveis mais elevados de } \\
\text { desemprego foram associados a maiores taxas de } \\
\text { suicídio. }\end{array}$ \\
\hline A9 & $\begin{array}{lrr}\text { Exposición } & \text { a } & \text { la } \\
\text { Violencia y } & \text { Riesgo } \\
\text { Suicida } & \text { en } \\
\text { Adolescentes } & \\
\text { Colombianos } & \\
\end{array}$ & 2018 & Terapia psicológica & $\begin{array}{l}\text { Foi encontrada associação bivariada com exposição } \\
\text { à violência doméstica. Em um modelo de regressão } \\
\text { logística apertada, o apoio pai/irmão/amigos é um } \\
\text { fator protetor para o risco suicida, enquanto o sexo } \\
\text { feminino exposição à violência doméstica e } \\
\text { dificuldades para acessar a educação são fatores de } \\
\text { risco. }\end{array}$ \\
\hline A10 & $\begin{array}{lr}\text { Caracterización } & \text { del } \\
\text { suicídio } & \text { en } \\
\text { adolescentes } & \text { del } \\
\text { Departamento } & \text { de } \\
\text { Quindío. Colombia, } \\
\text { 1989-2013 }\end{array}$ & 2017 & $\begin{array}{lr}\text { Revista } & \text { Habanera } \\
\text { de } & \text { Ciencias } \\
\text { Médicas } & \end{array}$ & $\begin{array}{l}\text { No período de } 1989 \text { a } 2013,158 \text { suicídios de } \\
\text { adolescentes foram registrados no Departamento de } \\
\text { Quindío. Assim, } 67,7 \% \text { eram do sexo masculino, } \\
82,9 \% \text { solteiros, } 39,9 \% \text { usavam enforcamento e } \\
\text { 13,3\% dos casos ocorreram em dezembro. A taxa } \\
\text { de suicídio foi de } 6,7 \text { por cem mil adolescentes - } \\
\text { 9,0 para homens e } 4,3 \text { para mulheres. O período de } \\
\text { cinco anos que registrou a maior taxa foi de } 1999 \text { a } \\
\text { 2003, com 10,0 suicídios por cem mil adolescentes. } \\
\text { Pelos municípios, o maior risco foi registrado em } \\
\text { Quimbaya e Pijao, com } 30,8 \text { e } 23,4 \text { suicídios por }\end{array}$ \\
\hline
\end{tabular}




\begin{tabular}{|c|c|c|c|c|}
\hline & & & & cem mil adolescentes, respectivamente. \\
\hline A11 & $\begin{array}{l}\text { Biting myself so I } \\
\text { don't bite the dust: } \\
\text { prevalence and } \\
\text { predictors of } \\
\text { deliberate self-harm } \\
\text { and suicide ideation in } \\
\text { Azorean youths }\end{array}$ & 2017 & $\begin{array}{l}\text { Brazilian Journal of } \\
\text { Psychiatry }\end{array}$ & $\begin{array}{l}\text { Aproximadamente } 30 \% \text { dos jovens relataram pelo } \\
\text { menos um comportamento de autolesão ou } \\
\text { autolesão não suicida, uma taxa duas vezes maiores } \\
\text { do que a maioria dos estudos realizados em } \\
\text { Portugal e em outros países europeus. Morder-se } \\
\text { foi a forma mais frequente de comportamentos de } \\
\text { autolesão ou autolesão não suicida, e os } \\
\text { comportamentos serviram para a regulação de } \\
\text { estados emocionais destrutivos. }\end{array}$ \\
\hline A12 & $\begin{array}{l}\text { Consumo de } \text { alcohol, } \\
\text { participación de los } \\
\text { padres, y otros } \\
\text { predictores de } \\
\text { en laicídio } \\
\text { boliviana }\end{array}$ & 2017 & $\begin{array}{ll}\text { Gaceta } & \text { Médica } \\
\text { Boliviana } & \end{array}$ & $\begin{array}{l}\text { Fatores de risco que se correlacionam } \\
\text { estatisticamente com o suicídio em adolescentes } \\
\text { escolares são os que seguem do mais alto ao menor: } \\
\text { ser vítima de bullying, uso de tabaco e/ou de álcool. } \\
\text { Os fatores protetores encontrados são: compreensão } \\
\text { dos problemas por parte dos pais, acompanhamento } \\
\text { dos pais no tempo livre das crianças, supervisão de } \\
\text { tarefas e atividade física. }\end{array}$ \\
\hline A13 & $\begin{array}{l}\text { Principales factores de } \\
\text { riesgo relacionados } \\
\text { con el intento suicida } \\
\text { en un grupo de } \\
\text { adolescentes }\end{array}$ & 2017 & $\begin{array}{l}\text { Revista Médica de } \\
\text { Santiago de Cuba- } \\
\text { MEDISAN }\end{array}$ & $\begin{array}{l}\text { Predominaram as mulheres } 73,5 \% \text {, com faixa etária } \\
\text { entre } 15 \text { a } 19 \text { anos } 60,9 \% \text {. Destaca-se a ingestão de } \\
\text { fármacos como o método mais utilizado em } 51,7 \% \text {. } \\
\text { Os principais fatores predisponentes foram: } \\
\text { histórico familiar hereditário } 19,5 \% \text {, violência } \\
\text { doméstica } 18,3 \% \text {, além de transtornos } \\
\text { comportamentais e depressão } 17,2 \% \text {. }\end{array}$ \\
\hline A14 & $\begin{array}{l}\text { Autoestima } \\
\text { Desesperanza En } \\
\text { Adolescentes De Una } \\
\text { Institución Educativa } \\
\text { Del Quindío }\end{array}$ & 2017 & Duazary & $\begin{array}{l}\text { Risco de suicídio ou grau "nenhum ou mínimo" de } \\
\text { desesperança foi encontrado em } 59,4 \% \text {, risco leve } \\
26,8 \% \text {, moderado } 12,9 \% \text { e alto de } 0,9 \% \text {. O risco } \\
\text { mínimo nas mulheres foi de } 33,5 \% \text { e alto de } 0,9 \% \text {, } \\
\text { enquanto nos homens o risco mínimo prevalece } \\
29,5 \% \text {, leve } 17 \% \text { e moderado } 7,1 \% \text {. A desesperança } \\
\text { é aumentada nos adolescentes por fatores } \\
\text { motivacionais e cognitivos que moldam a ideação } \\
\text { suicida e aumentam os riscos de suicídio. Baixa } \\
\text { estima não é um fator consistente na ideação } \\
\text { suicida. }\end{array}$ \\
\hline A15 & $\begin{array}{l}\text { Escala de avaliação de } \\
\text { humor para } \\
\text { adolescentes: } \\
\text { evidências de validade }\end{array}$ & 2016 & $\begin{array}{l}\text { Arquivos } \\
\text { Brasileiros } \\
\text { Psicologia }\end{array}$ & $\begin{array}{l}\text { Houve diferença significativa entre os sexos, em } \\
\text { relação à idade houve interações significativas no } \\
\text { fator mania e risco de suicídio. Os três fatores } \\
\text { apresentaram correlações significativas com as } \\
\text { escalas de depressão e de desesperança. }\end{array}$ \\
\hline A16 & $\begin{array}{l}\text { Comportamientos de } \\
\text { riesgo de suicídio y } \\
\text { calidad de vida, por }\end{array}$ & 2015 & $\begin{array}{l}\text { Ciência \& Saúde } \\
\text { Coletiva }\end{array}$ & $\begin{array}{l}\text { Análises separadas para mulheres e homens } \\
\text { mostraram os comportamentos de risco de suicídio } \\
\text { associados à qualidade de vida foi o }\end{array}$ \\
\hline
\end{tabular}




\begin{tabular}{|c|c|c|c|c|}
\hline & $\begin{array}{l}\text { género, en } \\
\text { adolescentes } \\
\text { mexicanos, estudiantes } \\
\text { de preparatória }\end{array}$ & & & $\begin{array}{l}\text { comportamento, para aqueles que sentiram-se } \\
\text { tristes ou sem esperança. Os modelos de regressão } \\
\text { mostram que alguns comportamentos de risco de } \\
\text { suicídio aumentam a chance de uma qualidade de } \\
\text { vida menor. }\end{array}$ \\
\hline A17 & $\begin{array}{l}\text { Suicídios en menores } \\
\text { de } 20 \text { años. México } \\
\text { 1998-2011 }\end{array}$ & 2015 & Salud Mental & $\begin{array}{l}\text { Na população de cinco a } 19 \text { anos, o suicídio foi } \\
\text { responsável por } 7 \% \text { das mortes por lesões causadas } \\
\text { externamente. O método mais utilizado foi o } \\
\text { enforcamento com } 75,7 \% \text {. As mulheres tiveram a } \\
\text { maior variação percentual, com } 6 \% \text {. O estado de } \\
\text { Hidalgo apresentou uma taxa maior, com } 17,2 \% \text {. } \\
\text { Em } 2011 \text {, a maior taxa de anos de vida perdida para } \\
\text { o suicídio ocorreu em Tabasco, com } 67 \% \text {. }\end{array}$ \\
\hline A18 & $\begin{array}{l}\text { Clinical } \\
\text { suicide ressment of } \\
\text { suicide attempters' } \\
\text { self-reported } \\
\text { intent: } \text { suicide } \\
\text { sectional study }\end{array}$ & 2019 & PLoS One & $\begin{array}{l}\text { O preditor mais forte da intenção suicida foi o mau } \\
\text { enfrentamento habitual, seguido de sérios } \\
\text { problemas financeiros. O preditor mais forte do } \\
\text { risco de suicídio foi esconder a tentativa seguida de } \\
\text { planejamento prévio. }\end{array}$ \\
\hline A19 & $\begin{array}{lr}\text { Suicide } & \text { risk in } \\
\text { adolescents } & \text { with fetal } \\
\text { alcohol } & \text { spectrum } \\
\text { disorders } & \end{array}$ & 2019 & $\begin{array}{ll}\text { Birth } & \text { Defects } \\
\text { Research } & \end{array}$ & $\begin{array}{l}\text { A prevalência de comportamentos suicidas nesta } \\
\text { amostra foi elevada, com } 35,2 \% \text { dos adolescentes } \\
\text { relatando incidências de ideação suicida, e } 13,0 \% \\
\text { relatando pelo menos uma tentativa grave de } \\
\text { suicídio no último ano. Esse achado contrasta com } \\
\text { os } 17,2 \% \text { e } 2,4 \% \text { para ideação e tentativas graves, } \\
\text { respectivamente, relatadas na população de } \\
\text { adolescentes dos EUA. Alarmantemente, } 29,2 \% \\
\text { dos homens com transtornos do espectro alcoólico } \\
\text { fetal relataram uma grave tentativa de suicídio. } \\
\text { Nenhuma mulher relatou tentativas. O número de } \\
\text { colocações domiciliares e a presença de transtorno } \\
\text { depressivo contribuíram para os desfechos do } \\
\text { estudo. }\end{array}$ \\
\hline A20 & $\begin{array}{l}\text { Psychopathology } \\
\text { profiles of acutely } \\
\text { suicidal adolescents: } \\
\text { Associations with } \\
\text { post-discharge suicide } \\
\text { attempts and } \\
\text { rehospitalization }\end{array}$ & 2017 & $\begin{array}{l}\text { Journal of Affective } \\
\text { Disorders }\end{array}$ & $\begin{array}{l}\text { A análise de perfil latente identificou quatro perfis } \\
\text { de psicopatologia: subclínico, principalmente } \\
\text { internalizado e moderadamente e severamente } \\
\text { desregulado. Na linha de base, os perfis diferem } \\
\text { pela história de autolesões não suicidas e múltiplas } \\
\text { tentativas de suicídio, bem como gravidade da } \\
\text { ideação suicida, desesperança, sintomas } \\
\text { depressivos, sintomas de ansiedade, abuso de } \\
\text { substâncias e comprometimento funcional. Os } \\
\text { perfis de desregulação previram tentativas de } \\
\text { suicídio dentro de } 3 \text { meses após a alta. O perfil } \\
\text { internalizador previu tentativas de suicídio e re- }\end{array}$ \\
\hline
\end{tabular}




\begin{tabular}{|c|c|c|c|c|}
\hline & & & & hospitalização aos 3 e 12 meses. \\
\hline A21 & $\begin{array}{l}\text { The Effects of the } \\
\text { Family Bereavement } \\
\text { Program to Reduce } \\
\text { Suicide Ideation } \\
\text { and/or Attempts of } \\
\text { Parentally Bereaved } \\
\text { Children Six and } \\
\text { Fifteen Years Later }\end{array}$ & 2016 & $\begin{array}{l}\text { Suicide Life Threat } \\
\text { Behav }\end{array}$ & $\begin{array}{l}\text { Os resultados indicam um efeito significativo do } \\
\text { FBP para reduzir a ideação e/ou tentativas de } \\
\text { suicídio na avaliação de seguimento de } 6 \text { e } 15 \text { anos. }\end{array}$ \\
\hline A22 & $\begin{array}{l}\text { Rehospitalization of } \\
\text { Suicidal Adolescents } \\
\text { in Relation to Course } \\
\text { of Suicidal Ideation } \\
\text { and Future Suicide } \\
\text { Attempts }\end{array}$ & 2016 & Psychiatr Services & $\begin{array}{l}\text { A re-hospitalização esteve associada ao maior risco } \\
\text { de tentativas de suicídio, acima dos efeitos das } \\
\text { covariáveis-chave. A re-hospitalização também } \\
\text { previu mudanças distintas nas trajetórias de ideação } \\
\text { suicida: Dentro dos grupos elevados e cronicamente } \\
\text { elevados, a re-hospitalização previu aumentos na } \\
\text { ideação durante o seguimento, com maior } \\
\text { magnitude para o grupo crônico. Em contrapartida, } \\
\text { a re-hospitalização esteve associada à diminuição } \\
\text { da ideação suicida no grupo subclínico. }\end{array}$ \\
\hline A23 & $\begin{array}{l}\text { Investigating the role } \\
\text { of hopelessness in the } \\
\text { relationship between } \\
\text { PTSD symptom } \\
\text { change and suicidality }\end{array}$ & 2018 & $\begin{array}{l}\text { Journal of Affective } \\
\text { Disorders }\end{array}$ & $\begin{array}{l}\text { A desesperança pré-tratamento emergiu como um } \\
\text { moderador significativo, de tal forma que as } \\
\text { reduções gerais de sintomas de transtorno de } \\
\text { estresse pós-traumático estavam relacionadas a } \\
\text { reduções globais na probabilidade de futura } \\
\text { tentativa de suicídio entre aqueles que estavam ou } \\
\text { acima da média amostral de desesperança pré- } \\
\text { tratamento. }\end{array}$ \\
\hline A24 & $\begin{array}{l}\text { Is socioeconomic } \\
\text { position associated } \\
\text { with risk of attempted } \\
\text { suicide in rural Sri } \\
\text { Lanka? A cross- } \\
\text { sectional study of } 165 \\
000 \text { individuals }\end{array}$ & 2017 & BMJ Open & $\begin{array}{l}\text { Os domicílios relataram } 398 \text { tentativas de suicídio } \\
\text { no ano anterior ( } 239 \text { por } 100 \text { mil). Em nível } \\
\text { individual, os trabalhadores assalariados diários } \\
\text { estavam em maior risco de tentativa de suicídio em } \\
\text { comparação com os agricultores. As associações } \\
\text { mais fortes foram com baixos níveis de } \\
\text { escolaridade, com associação mais forte em homens } \\
\text { do que em mulheres. }\end{array}$ \\
\hline A25 & $\begin{array}{l}\text { Suicide and Other- } \\
\text { Cause Mortality after } \\
\text { Early Exposure to } \\
\text { Smoking and Second } \\
\text { Hand Smoking: A } 12- \\
\text { Year Population- } \\
\text { Based } \quad \text { Follow-Up } \\
\text { Study }\end{array}$ & 2015 & PLoS One & $\begin{array}{l}\text { Os participantes que fumavam, apresentaram } \\
\text { mortalidade por suicídio até seis vezes maior do } \\
\text { que aqueles que não fumaram, bem como maior } \\
\text { mortalidade natural. Após o controle de gênero, } \\
\text { idade, escolaridade parental, asma, rinite alérgica e } \\
\text { consumo de álcool, as razões de risco ajustadas } \\
\text { para suicídio foram de fumantes atuais, e } \\
\text { respectivamente em adolescentes expostos ao } \\
\text { tabaco. }\end{array}$ \\
\hline
\end{tabular}


Fonte: Dados da Pesquisa (2020).

Em relação aos artigos selecionados nesta revisão, destaca-se o ano de 2017 com nove publicações, seguido por 2019 com cinco, 2016 e 2018 com quatro manuscritos anuais correlacionadas com a temática, e por fim 2015 com três artigos. Dentre os países com maior número de estudos desenvolvidos encontram-se o México e a Colômbia com quatro, e na sequência Brasil e Estados Unidos com três estudos, as demais pesquisas foram realizadas em outros países.

Referente às tentativas de suicídio, os achados desta revisão revelam que adolescentes com depressão ou com fatores psiquiátricos prévios podem cometer mais suicídio do que adolescentes que não possuem este histórico [A7, A11, A13, A14, A15, A19], sendo a depressão um significativo fator de risco no aumento da ideação suicida [A13, A14, A19].

O artigo [A19] remete um estudo realizado com 54 adolescentes entre 13 a 18 anos e aponta que 36,8\% dos jovens que apresentavam critérios para transtornos depressivos relataram ideação suicida, sendo que na amostra masculina $42,9 \%$ referiram graves tentativas de suicídio anteriores.

Para além disso, outro fator relevante para a tentativa de suicídio relaciona-se com a presença de sintomas sugestivos de transtornos alimentares, para o qual foi evidenciada maior prevalência na população feminina. De acordo com estudo [A4] $17,8 \%$ dos adolescentes que apresentam tais sintomatologias pensam no suicídio como uma alternativa e $15,38 \%$ desse total afirmam tentativa prévia de suicídio.

As publicações [A5, A14, A16, A23] sinalizam que os dilemas da adolescência somados aos acontecimentos de vida negativos e à desesperança possuem correlação com o aumento no risco de suicídio em adolescentes, sendo que a baixa estima e a baixa percepção de razões para viver possuem associação com os índices mais elevados da ideação suicida [A5], assim como as formas severas de autocríticas, ansiedade e estresse [A11].

Outro fator expressivo nos índices das tentativas de suicídio são as relações familiares, as quais são responsáveis pela influência direta no ambiente em que os adolescentes estão inseridos. Dados apresentados no artigo [A7] realizado com estudantes de escolas públicas e privadas, com 26.503 alunos, composto em sua maioria pelo sexo masculino com idades inferiores a 15 anos, uma relação familiar ruim gera um aumento de 2,5 na prevalência de tentativas de suicídio, em comparação àqueles que vivem de forma favorável. A ruim relação com os pais atua como fator de crescimento das tentativas suicidas, aumentando em até três vezes sua prevalência. Famílias disfuncionais, relações intrafamiliares fracas e desfavoráveis também agem como fatores de risco para o aumento de tentativas de suicídio em adolescentes [A13, A14].

Dentre os elementos que se correlacionam como o aumento do risco de suicídio na população adolescente foi evidenciado pelos artigos [A3, A9] a exposição dos adolescentes à violência, seja em ambiente doméstico, escolar e nas ruas. De acordo com estudo [A3], realizado com informações de 21.509 adolescentes entre 10 a 19 anos, a cada 100 adolescentes vítimas de violência sexual 8 informam tentativa de suicídio no ano anterior à pesquisa.

Conforme o demonstrado no artigo [A8], a diminuição no Produto Interno Bruto PIB, acrescido de aumento nas taxas de desemprego da população, associa-se com maiores taxas de suicídio, em que a desigualdade social apresenta vinculação ao suicídio na faixa etária dos 15 aos 19 anos. Em paralelo a esse dado, outros estudos [A6, A18, A24] apresentam conexão com as chances de intenção de suicídio a problemas socioeconômicos relacionados às questões financeiras como o desemprego e/ou o emprego instável. Achados sinalizam também que os problemas financeiros foram causadores de aumento de 4,39 nas chances de intenção suicida em adolescentes [A18].

Ao passo que problemas financeiros se correlacionam com aumento nas taxas de ideação suicida os fatores educacionais como a baixa escolaridade, dificuldades de ingresso na educação também apresentam associação semelhante [A3, A7, A9, A24]. A defasagem educacional aumentou a tentativa de suicídio em adolescentes com faixa etária dos 10 aos 19 anos, conforme o evidenciado no estudo [A3], sendo que dos adolescentes que apresentam risco de suicídio, uma porcentagem de 
27,6\% encontra dificuldade em continuar seus estudos [A9].

Também foi expresso correlação com uso de drogas lícitas e ilícitas a fatores de risco para o suicídio em adolescentes [A3, A7, A12, A25]. Em relação ao fumo, de acordo com [A3] consumir cinco maços de cigarro durante a vida faz com que a prevalência de suicídio se torne o dobro comparado aos não fumantes, e aos que ingerem álcool essa taxa aumenta para três vezes em comparação aos que não fumam.

No que diz respeito ao suicídio, este é mais presente entre a população masculina [A1, A8, A10, A17], em que a idade de maior prevalência foi entre os 15 a 19 anos [A8, A17]. Dentre as mulheres a ideação e a tentativa suicida são verificadas em maiores índices [A2, A3, A7, A13], o suicídio é observado em faixa etária feminina mais jovem entre os 10 a 16 anos em comparação ao grupo de 17 a 19 anos [A3].

A respeito das estratégias utilizadas observa-se a utilização de métodos mais letais na população masculina como o enforcamento, envenenamento por pesticidas e armas de fogo [A1, A10, A17] sendo que na população feminina o método utilizado observado no estudo foi a ingesta de psicofármacos [A10, A13]. O suicídio por meio do enforcamento também se faz presente nas mulheres [A1, A10] de acordo com estudo realizado a partir da análise de 158 adolescentes que cometeram suicídio no Departamento de Quindío, 31,4\% das mulheres utilizaram o enforcamento como método de suicídio. Com relação ao local de escolha para a consumação do suicídio o domicílio é o local de maior evidência [A10, A17, A18].

O estudo [A18] o qual baseia-se em uma análise retrospectiva de registros hospitalares de pacientes hospitalizados por tentativa de suicídio de janeiro de 2004 a dezembro de 2006, aponta que ocultação da tentativa de suicídio bem como planejamento prévio deste ato, faz com que as chances de serem avaliados como suicídio de alto risco aumentem em números consideráveis.

Diante disso, os resultados obtidos demonstram que o suicídio é algo multifatorial e encontra-se correlacionado com aspectos sociais, biológicos e psicológicos de cada adolescente, em que questões relacionadas a exposição à violência, relações familiares conturbadas, acontecimentos de vida negativos, desigualdades sociais, problemas educacionais, depressão e uso de drogas lícitas e ilícitas atuam como fatores de risco para o suicídio em adolescentes.

\section{Discussão}

A partir dos resultados dessa revisão narrativa, observou-se que adolescer é um processo permeado por expectativas sociais, em que o indivíduo enfrenta um processo de maturação biológica, psicológica e emocional, sendo um período de aumento das responsabilidades sociais, familiares e profissionais. Tais aspectos somados à necessidade de busca por um papel social e as pressões sociais vivenciadas, podem tornar-se fatores desencadeantes de sofrimento psíquico no adolescente, fazendo com que sentimentos de desesperança, inutilidade e insuficiência venham à tona e atuem como agentes geradores de estresse e ansiedade, podendo resultar em comportamentos e ideações suicidas (Meine; Cheiram \& Jaeger, 2019).

Dentre esses, a desesperança pode ser definida como uma construção transitória que envolve sentimentos negativos e a ausência de uma perspectiva para o futuro, podendo ser relacionada positivamente com a ideação suicida (Chang, 2017). Segundo estudos a realização de planejamento ou tentativas de suicídio prévios correlacionou-se com a apresentação de sentimento de tristeza e desespero em adolescentes, fato este que contribuiu para diminuição na qualidade de vida destes indivíduos (Hidalgo-Rasmussen \& Martín, 2015; James, et al., 2017).

De acordo com pesquisa, realizada com 971 estudantes matriculados em um colégio dependente da Universidad Nacional Autónoma de México, foi possível identificar relação estatística significativa nos sexos masculino e feminino com relação ao risco de cometer suicídio, medido por meio do prognóstico da Escala de Desesperança de Beck. Nesse contexto, o risco é encontrado quando na infância e adolescência o indivíduo conviveu com apenas um dos pais ou outro responsável, quando há ausência de amigos com quem contar, estabelecimento de muitas ou nenhuma regra sobre a criança e/ou 
adolescente, bem como apresentar proximidade com quem suicidou-se ou tentou se suicidar (Osnaya \& Pérez, 2010).

Correlacionado com o aumento do risco de suicídio em adolescentes, encontram-se também os acontecimentos de vida considerados negativos, os quais recebem essa classificação quando há a ocorrência de eventos traumáticos, como a morte ou doença de familiares e/ou amigos próximos, problemas familiares, bem como as alterações nas relações afetivas como o término de relações amorosas, dentre outros (Sobrinho \& Campos, 2016).

A forma como o adolescente percebe esses acontecimentos influencia diretamente em sua resposta, podendo este manter seu equilíbrio emocional por meio da adaptação a possíveis mudanças impostas, ou então tais eventos podem ter um impacto desestruturante, causando um desiquilíbrio psicológico em resposta a um aumento de stress, propiciando assim o desenvolvimento de algumas patologias, como a depressão (Sobrinho \& Campos, 2016).

Desta forma, percebe-se os desafios que permeiam a adolescência associados a padrões e expectativas sociais, parecem influenciar a forma como o indivíduo compreende os acontecimentos que vivencia. Isso faz com que essas situações estressoras contribuam para o desenvolvimento da depressão em adolescentes, sendo esta a patologia mais frequente entre os jovens (Meine; Cheiram \& Jaeger, 2019).

A depressão pode ser descrita pela incapacidade de realizar atividades diárias e perda de interesse por atividades que antes traziam prazer, somada ao sentimento de tristeza constante, por pelo menos duas semanas (OPAS \& OMS, 2017). Para além disso, há aspectos que contribuem para o desenvolvimento da depressão, dentre eles é possível citar doenças orgânicas, pressão social muitas vezes associada à discriminação, fatores hereditários, conflito familiar, exposição a violências, abandono, dentre outros (Meine; Cheiram \& Jaeger, 2019).

Estudo realizado com 911 alunos entre 14 a 18 anos, matriculados em uma instituição pública do estado de Jalisco demonstrou que 33,4\% dos participantes apresentaram ideação suicida, sendo a depressão fator com associação moderada direta com o suicídio (Reynoso, et al., 2019). Este dado corrobora com o resultado evidenciado em pesquisa desenvolvida com 1538 estudantes de 15 a 19 anos de idade, a qual aponta que a depressão está presente em 67,3\% das pessoas que havia tentado suicídio e em $81.1 \%$ dos que apresentaram ideações suicidas (Rodríguez et al., 2012).

No entanto, o diagnóstico de transtornos depressivos em adolescentes por vezes é complexo e trabalhoso, devido à associação dos sintomas depressivos manifestados com rebeldia e indisciplina, ocasionado pela falta de conhecimento a respeito de tal patologia (Meine \& Cheiram \& Jaeger, 2019). Para além disso, a depressão faz com que o adolescente se torne mais recluso e quieto, dificultando, assim, a comunicação verbal e emocional entre esta população e seus responsáveis, o que contribui para tornar essa relação mais complicada e problemática (Zerbetto, et al., 2018).

Os problemas no diálogo e nas relações familiares influenciam diretamente no comportamento destes indivíduos, visto que a família é o primeiro espaço de interação social responsável por oferecer a base para o desenvolvimento do adolescente. De acordo com estudo realizado com 185 alunos de 12 a 15 anos de idade, os problemas familiares e de relacionamento com os pais apresentaram relação com o aumento do risco de suicídio em adolescentes, sendo que a violência familiar foi descrita como a segunda principal causa de suicídio, de acordo com a percepção dos participantes (Garza - Sánchez; Saucedo \& García, 2019).

Estudo desenvolvido com 23 adolescentes com tentativa prévia de suicídio, demonstra que 39,1\% dos participantes apresentava família disfuncional; destes, 52,2\% severamente disfuncionais, o que significa que a maioria das famílias tem processos relacionais problemáticos e o ambiente cotidiano não se manifesta com harmonia, afetividade, boa comunicação e bom desempenho de papel (Mirabal \& Bernal, 2015).

Alguns fatores externos também são responsáveis por influenciar negativamente as relações familiares tornando-as complicadas e desgastadas. Dentre eles é possível citar os problemas socioeconômicos, os quais atuam como agentes causadores do aumento do risco de suicídio em adolescentes, devido ao acréscimo da carga de estresse e ansiedade nesta 
população. Segundo pesquisa desenvolvida com adolescentes com tentativas de suicídio, $87 \%$ do total apresentaram renda econômica insuficiente (Mirabal \& Bernal, 2015).

Os problemas na comunicação e no relacionamento familiar, acrescidos dos problemas financeiros e sociais, são responsáveis por gerar sentimentos negativos nos adolescentes os distanciando, assim, do seu seio familiar. Esse fato faz com que essa população busque o prazer imediato a fim de aliviar a angústia sentida, o que os torna vulneráveis a diversos agravos, pois os adolescentes, por vezes, optam por sentir essa satisfação momentânea com o uso de drogas lícitas e ilícitas (Bittencourt; Garcia \& Goldim, 2015).

Fatores individuais, familiares, escolares e sociais exercem influência negativa no uso de drogas por adolescentes (Bittencourt, Garcia \& Goldim, 2015). Segundo pesquisa transversal realizada com 229 adolescentes usuários de substâncias psicoativas, do total 56,8\% relataram morar com algum parente que faz uso de algum tipo de droga, e cerca de $62,9 \%$ apresentavam evasão escolar. Ainda, de acordo com o estudo, as drogas mais utilizadas foram a maconha, seguida pelo crack, cocaína, álcool e tabaco (Targino \& Hayasida, 2018).

O uso de substâncias psicoativas expõe os adolescentes ao risco de dependência, o que irá comprometer a realização de suas tarefas cotidianas, bem como seu relacionamento familiar, fragilizando-os e tornando-os susceptíveis ao suicídio. Segundo pesquisa exploratória descritiva e corte transversal realizado com 816 estudantes de 13 a 18 anos foi verificada associação positiva do uso de drogas com a ideação suicida (Barros, Pichelli \& Ribeiro, 2017).

\section{Considerações Finais}

A realização deste estudo possibilitou observar que os adolescentes estão vulneráveis aos diversos agravos que podem fragilizar sua saúde mental, o que somado aos fatores de risco de origem biológica, econômica, psicológica e social, atuam como agentes para o desenvolvimento de ideações suicidas e/ou tentativas de suicídio.

Esta revisão narrativa pode contribuir para o desenvolvimento da visão integral dos profissionais de enfermagem a respeito da saúde do adolescente, em especial a saúde mental, no âmbito da Atenção Primária à Saúde por meio do Programa Saúde na Escola, com ações que priorizam a saúde de adolescentes e jovens. O enfoque refere-se à promoção da saúde de escolares a fim de contribuir para a identificação dos fatores de risco e prevenção do suicídio, bem como para o campo de formação em Enfermagem, visto que essa temática deve ser abordada nos currículos de graduação.

Como limitações do estudo destacam-se artigos científicos que não convergiam com a temática, o que reforça a necessidade da construção de resumos estruturados e a escolha de descritores que façam referência aos manuscritos publicados. Todavia, as limitações para o levantamento dos manuscritos não implicaram no objetivo proposto nesta revisão narrativa.

Por fim, sugere-se a realização de estudos de campo com a população adolescente, que abordem as questões relacionadas ao suicídio, ideações suicidas e tentativas de suicídio, abrangendo, assim, as questões de saúde mental deste grupo extremamente vulnerável.

\section{Referências}

Abreu, T. O., \& Souza, M. B. (2017). A influência da internet nos adolescentes com ações suicidas. Revista Sociais \& Humanas. 30 (1), 158-173. $10.5902 / 2317175825868$.

Alarcão, A. C. et al. (2020). Suicide mortality among youth in southern Brazil: a spatiotemporal evaluation of socioeconomic vulnerability. Braz. J. Psychiatry [online] 42 (1), 46-53. 10.1590/1516-4446-2018-0352.

Ardiles-Irarrazabal, R. A. et al. (2018). Riesgo de suicidio adolescente en localidades urbanas y rurales por género, región de Coquimbo, Chile. Aquichan. 18 (2), 160-170. 10.5294/aqui.2018.18.2.4.

Baquero, L C. M. et al. (2017). Association between symptoms of anorexia and bulimia nervosa and suicidal behavior in school children of Boyacá, Colombia. Act.Colom.Psicol 20 (2), 189-199. 10.14718/ACP.2017.20.2.9. 
Barros, P. D. Q., \& Pichelli, A. A. W. S., \& Ribeiro K. C. S. (2017). Associação entre o consumo de drogas e a ideação suicida em adolescentes. Mental [online]. 11 (21), 304-320. http://pepsic.bvsalud.org/scielo.php?script=sci_arttext\&pid=S1679-44272017000200002\&lng=pt\&tlng=pt.

Berona, J., \& Horwitz, A. G., \& Czyz, E.K., \& King, C.A. (2020). Psychopathology profiles of acutely suicidal adolescents: Associations with post-discharge suicide attempts and rehospitalization. J Affect Disord. 209, 97-104. 10.1016/j.jad.2016.10.036.

Bittencourt, A. L. P., \& Garcia, L. F., \& Goldim, J. R. (2015). Vulnerable adolescence: bio-psychosocial factors related to drug use. Rev. Bioét. [online]. 23 (2), 308-316. 10.1590/1983-80422015232070.

Boffa, J.W., \& King, S.L., \& Turecki, G., \& Schmidt, N.B. (2018) Investigating the role of hopelessness in the relationship between PTSD symptom change and suicidality. J Affect Disord. 225, 298-301. 10.1016/j.jad.2017.08.004

Braga, D. É. M., \& Marques, J. C., \& Alves, P. C. (2017). Suicídio na adolescência: uma revisão integrativa de literatura. In: IX Encontro de Pesquisa e Extensão da Faculdade Luciano Feijão. ARTIGO_SUICIDIO_NA_ADOLESCENCIA_UMA_REVISAO_INTEGRATIVA_DE_LITERATURA.pdf (flucianofeijao.com.br).

Bras, M., \& Jesus, S., \& Carmo, C. (2016). Fatores psicológicos de risco e protetores associados à ideação Suicida em Adolescentes. Psic., Saúde \& Doenças [online].17 (2), 132-149. 10.15309/16psd170203.

Brasil. Presidência da República. (1990). Lei 8069, de 13 de Julho de 1990. Estatuto da Criança e do Adolescente. http://www.planalto.gov.br/.

Brum, C.N. et al. (2015). Revisão narrativa da literatura: aspectos conceituais e metodológicos na construção do conhecimento da enfermagem. In: Lacerda, M.R., \& Costenaro, R.G.S.Metodologias da pesquisa para a enfermagem e saúde. Moriá.

Caballero, M. A. et al. (2017). Principales factores de riesgo relacionados con el intento suicida en un grupo de adolescentes. MEDISAN [online]. 21 (2), 154160. http://scielo.sld.cu/scielo.php?script=sci_arttext\&pid=S1029-30192017000200004\&lng=es\&tlng=es.

Cardozo, R. K. A., \& Alvarez, A. A. (2017). Consumo de alcohol, participación de los padres, y otros predictores de suicidio en la juventud boliviana. Gac Med Bol. 40 (1), 29-34. http://www.scielo.org.bo/scielo.php?script=sci_arttext\&pid=S1012-29662017000100007\&lng=es\&tlng=es.

Carvalho, C. B. et al. (2017). Biting myself so I don't bite the dust: prevalence and predictors of deliberate self-harm and suicide ideation in Azorean youths. Rev. Bras. Psiquiatr. 39 (3), 252-262. 10.1590/1516-4446-2016-1923.

Castelar, M., \& Daltro, M. R., \& Barreto, J. D. (2019). A caminho do LILACS. Rev. Psicol. Divers. Saúde 8, (1), 5-7. 10.17267/2317-3394rpds.v8i1.2308.

Cervantes, C. A. D., \& Contreras, M. L. (2019). Intento de suicidio en adolescentes: Factores asociados. Rev. chil. pediatr. 90 (6), 606-616. 10.32641/rchped.v90i6.1012.

Chang, E.C. (2017). Hope and hopelessness as predictors of suicide ideation in Hungarian college students. Death Studies 41, (7), 455-460. $10.1080 / 07481187.2017 .1299255$.

Chen, V.C. et al. (2015). Suicide and Other-Cause Mortality after Early Exposure to Smoking and Second Hand Smoking: A 12-Year Population-Based Follow-Up Study. PLoS One. 10 (7). 10.1371/journal.pone.0130044.

Choo, C.C., \& Harris, K.M., \& Chew, P.K.H., \& Ho, R.C. (2019). Clinical assessment of suicide risk and suicide attempters' self-reported suicide intent: A cross sectional study. PLoS One. 14 (7). 10.1371/journal.pone.0217613.

Contreras, M. L., \& Cervantes, C. A. D. (2018). Adolescentes en riesgo: factores asociados con el intento de suicidio en México. Rev. Gerenc. Polit. Salud. 17, (34), 1-12. 10.11144/Javeriana.rgsp17-34.arfa.

Costa, P. H. A. et al. (2015). Desatando a trama das redes assistenciais sobre drogas: uma revisão narrativa da literatura. Ciência \& Saúde Coletiva [online]. 20 (2), 395-406. 10.1590/1413-81232015202.20682013.

Couto, V. V. D., \& Tavares, M. S. A. (2016). Apego e risco de suicídio em adolescentes: estudo de revisão. Rev. SPAGESP. 17 (2), 120-136.: http://pepsic.bvsalud.org/scielo.php?script=sci_arttext\&pid=S1677-9702016000200010\&lng=pt.

Czyz, E.K., \& Berona, J., \& King, C.A. (2016). Rehospitalization of Suicidal Adolescents in Relation to Course of Suicidal Ideation and Future Suicide Attempts. Psychiatr Serv. 67 (3), 332-338. 10.1176/appi.ps.201400252.

Gadêlha, L. N., \& Gonçalves, F. M. S. (2017). A adolescência e a responsabilidade social. Psicologia.pt - O Portal dos Psicólogos. A adolescência e a responsabilidade social (psicologia.pt).

Garza - Sánchez, R. I., \& Saucedo L. K. C., \& García, S. A. C. (2019). Estructura familiar, ideación suicida y desesperanza en el adolescente. Psicología desde el Caribe 36 (2), 228-247. 10.14482/psdc.36.2.616.8.

Gerstner, R. M. F. et al. (2018). Epidemiología del suicidio en adolescentes y jóvenes en Ecuador. Revista Panamericana de Salud Pública [online] 42. 10.26633/RPSP.2018.100.

Hidalgo-Rasmussen, C., \& Martín, A. H.-S. (2015). Comportamientos de riesgo de suicidio y calidad de vida, por género, en adolescentes mexicanos, estudiantes de preparatoria. Ciênc. saúde coletiva. 20 (11), 3437-3445. 10.1590/1413-812320152011.18692014.

Jaen-Varas, D. et al.(2019). The association between adolescent suicide rates and socioeconomic indicators in Brazil: a 10-year retrospective ecological study. Braz. J. Psychiatry. 41 (5), 389-395.10.1590/1516-4446-2018-0223.

James, S. et al. (2017). The association between adolescent risk behaviours and feelings of sadness or hopelessness: a cross-sectional survey of South African secondary school learners. Psychology, Health \& Medicine. 22 (7), 778-789. 10.1080/13548506.2017.1300669. 
Knipe, D. W. et al. (2017). Is socioeconomic position associated with risk of attempted suicide in rural Sri Lanka? A cross-sectional study of 165000 individuals. BMJ Open. 7 (3). Retrieved from: https://bmjopen.bmj.com/content/7/3/e014006.

Medina-Pérez, O. A, \& Ospina-Sanchez, S. M., \& Cardona-Duque, D. V. (2017). Caracterización del suicidio en adolescentes del Departamento de Quindío. Colombia, 1989-2013. Rev haban cienc méd. 16 (5), 784-795. http://scielo.sld.cu/scielo.php?script=sci_arttext\&pid=S1729-519X2017000500011.

Medina-Pérez, O. A, \& Ospina-Sanchez, S. M., \& Cardona-Duque, D. V. (2017). Caracterización del suicidio en adolescentes del Departamento de Quindío. Colombia, 1989-2013. Rev haban cienc méd 16 (5), 784-795. http://scielo.sld.cu/scielo.php?script=sci_arttext\&pid=S1729-519X2017000500011.

Meine, I. R., \& Cheiram, M. C., \& Jaeger, F. P. (2019) Depressão e suicídio: o adolescente frente a fatores de risco socioculturais. Res., Soc. Dev. 8 (12). https://doi.org/10.33448/rsd-v8i12.1882.

Ministério da Saúde. (2017). Boletim Epidemiológico das tentativas e óbitos por suicídio no Brasil e a rede de atenção à saúde. Suicídio. Saber, agir e prevenir. 48 (30), 1-14. https://crianca.mppr.mp.br/pagina-2149.html.

Mirabal, L. C. H., \& Bernal, C. I. L. (2015). Principales características psicosociales de adolescentes con intento suicida. Revista Cubana de Medicina General Integral 31 (2), 182-189. http://scielo.sld.cu/scielo.php?script=sci_arttext\&pid=S0864-21252015000200007.

O'Connor, M. J., \& Portnoff, L. C. \& Lebsack-Coleman M., \& Dipple, K. M. (2019). Suicide risk in adolescents with fetal alcohol spectrum disorders. Birth Defects Res. 111 (12), 822-828. 10.1002 / bdr2.1465.

Organização Mundial da Saúde. Organização Panamericana de Saúde. (2016). Prevención de la conducta suicida. Washington, DC: OMS, OPAS. https://www.coursehero.com/file/77103361/Prevencion-de-la-conducta-suicida-OMS-OPSpdf/.

Organização Mundial da Saúde. Saúde para os adolescentes do mundo: uma segunda chance na segunda década. Genebra: Organização Mundial da Saúde, (2014). https://doi.org/10.1590/0102-311X00125018.

Organização Pan-Americana da Saúde (OPAS), \& Organização Mundial da Saúde (OMS). (2017). Depressão: o que você precisa saber. https://www.paho.org/bra/index.php?option=com_content\&view=article\&id=5372:depressaoo-que-voce-precisa-saber\&Itemid=822.

Osnaya, M. C., \& Pérez, J. C. R. (2010). La desesperanza de riesgo en jóvenes mexicanos y aspectos sociodemográficos asociados: diferencias por sexo. Psicología y Salud [online] 20 (2), 195-201. https://doi.org/10.25009/pys.v20i2.602.

Packer, A. L. et al. (1998). SciELO: uma metodologia para publicação eletrônica. Ci. Inf. 27 (2). https://doi.org/10.1590/S0100-19651998000200001.

Reppold, C. T., \& Gurgel, L. G., \& Hutz, C. S. (2016). Escala de avaliação de humor para adolescentes: evidências de validade. Arq. bras. psicol. [online] 2016, 68 (2), 21-30. http://pepsic.bvsalud.org/scielo.php?script=sci_arttext\&pid=S1809-52672016000200003.

Reynoso, O. et al. (2019). Modelo explicativo y predictivo de la ideación suicida en una muestra de bachilleres mexicanos. Psicología desde el Caribe [online] 36 (1), 82-100. 2011-7485-psdc-36-01-82.pdf (scielo.org.com).

Ricci, B. B. et al. (2016). Suicídio no brasil entre jovens e adolescentes. In: $16^{\circ}$ Congresso Nacional de Iniciação Científica. trabalho-1000022470.pdf (conicsemesp.org.br).

Rodríguez, M, J. C. et al. (2012). Depresión y comportamiento suicida en estudiantes de educación media superior en Sonora. Salud Mental [online] 35 (1), 45-50. http://www.scielo.org.mx/scielo.php?script=sci_arttext\&pid=S0185-33252012000100007\&lng=es.

Rosi, L. M. et al. (2019). Crise e saúde mental na adolescência: a história sob a ótica de quem vive. Cadernos de Saúde Pública. 35. https://doi.org/10.1590/0102-311X00125018.

Sadock, B. J., \& Sadock, V. A. (2017). Compêndio de psiquiatria: ciências do comportamento e psiquiatria clínica. (9a ed.), Artes Médicas.

Sanchez-Cervantes, F. S., \& Serrano-Gonzalez, R. E, \& Marquez-Caraveo, M. E. (2015). Suicidios en menores de 20 años. México 1998-2011. Salud Ment. 38 (5), 379-389. 10.17711/SM.0185-3325.2015.051.

Sandler, I., \& Tein, J. Y., \& Wolchik, S., \& Ayers, T. S. (2016). The Effects of the Family Bereavement Program to Reduce Suicide Ideation and/or Attempts of Parentally Bereaved Children Six and Fifteen Years Later. Suicide Life Threat Behav.46 Suppl 1(Suppl 1): S32-S38. https://doi.org/10.1111/sltb.12256.

Senna, S. R. C. M., \& Dessen, M. A. (2015). Reflexões sobre a saúde do adolescente brasileiro. Psic., Saúde \& Doenças 16 (2), $217-229$. http://www.scielo.mec.pt/scielo.php?script=sci_arttext\&pid=S164500862015000200008\&lng=pt. http://dx.doi.org/10.15309/15psd160208.

Silva, J. F. et al. (2019). Adolescência e saúde mental: a perspectiva de profissionais da Atenção Básica em Saúde. Interface. 23: e180630. https://doi.org/10.1590/Interface.180630.

Silva, L. L. T. et al. (2015). O suicídio na adolescência nas publicações da enfermagem brasileira: revisão integrativa da literatura. R. Enferm. Cent. O. Min. 5 (3). https://doi.org/10.19175/recom.v5i3.767.

Sistema de Informação de Agravos de Notificação - SINAN. (2020) TabNet Win32 3.0: VIOLÊNCIA DOMÉSTICA, SEXUAL E/OU OUTRAS VIOLÊNCIAS - Brasil (datasus.gov.br).

Sobrinho, A. T., \& Campos, R. C. (2016). Perceção de acontecimentos de vida negativos, depressão e risco de suicídio em jovens adultos. Aná. Psicológica. 34(1), 47-59. http://dx.doi.org/10.14417/ap.1061.

Suarez, Y. et al. (2018). Exposición a la Violencia y Riesgo Suicida en Adolescentes Colombianos. Ter Psicol. 36 (2), 101-111. https://scielo.conicyt.cl/pdf/terpsicol/v36n2/0718-4808-terpsicol-36-02-00101.pdf. 
Research, Society and Development, v. 10, n. 2, e54410212730, 2021

(CC BY 4.0) | ISSN 2525-3409 | DOI: http://dx.doi.org/10.33448/rsd-v10i2.12730

Targino, R., \& Hayasida, N. (2018). Risco e proteção no uso de drogas: revisão da literatura. Psicologia, Saúde \& Doenças 19 (3), $724-742$. http://dx.doi.org/10.15309/18psd190320.

Zerbetto, S. R. et al. (2018). As relações familiares com adolescentes usuários de substâncias psicoativas: percepção dos pais. Rev. Eletr. Enf 20. https://doi.org/10.5216/ree.v20.46353. 